\title{
Neuroinflammatory Cytokine Response, Neuronal Death and Microglial Proliferation in the Hippocampus of Rats During the Early Period After Lateral Fluid-Percussion-Induced Traumatic Injury of the Neocortex
}

Ilia G. Komoltsev

Institute of Higher Nervous Activity and Neurophysiology RAS, Moscow

Liya V. Tretyakova

Institute of Higher Nervous Activity and Neurophysiology RAS, Moscow

Stepan 0. Frankevich

Institute of Higher Nervous Activity and Neurophysiology RAS, Moscow

Natalia I. Shirobokova

Institute of Higher Nervous Activity and Neurophysiology RAS, Moscow

Aleksandra A. Volkova

Institute of Higher Nervous Activity and Neurophysiology RAS, Moscow

Alexey V. Butuzov

Institute of Higher Nervous Activity and Neurophysiology RAS, Moscow

Margarita R. Novikova

Institute of Higher Nervous Activity and Neurophysiology RAS, Moscow

Alexey A. Kvichansky

Institute of Higher Nervous Activity and Neurophysiology RAS, Moscow

\section{Yulia V. Moiseeva}

Institute of Higher Nervous Activity and Neurophysiology RAS, Moscow

Mikhail V. Onufriev

Institute of Higher Nervous Activity and Neurophysiology RAS, Moscow

Alexey P. Bolshakov

Institute of Higher Nervous Activity and Neurophysiology RAS, Moscow

Natalia Gulyaeva ( $\nabla$ nata_gul@ihna.ru )

Institute of higher Nervous Activity and Neurophysiology RAS https://orcid.org/0000-0002-5380-7954

Research Article 
Keywords: traumatic brain injury, hippocampus, neuroinflammation, microglia, neurodegeneration, distant damage.

Posted Date: September 29th, 2021

DOI: https://doi.org/10.21203/rs.3.rs-762477/v2

License: (1) This work is licensed under a Creative Commons Attribution 4.0 International License. Read Full License

Version of Record: A version of this preprint was published at Molecular Neurobiology on December 2nd, 2021. See the published version at https://doi.org/10.1007/s12035-021-02668-4. 


\section{Abstract}

Time course of changes in neuroinflammatory processes in the dorsal and ventral hippocampus was studied during the early period after lateral fluid-percussion-induced neocortical traumatic brain injury (TBI) in the ipsilateral and contralateral hemispheres. In the ipsilateral hippocampus, neuroinflammation (increase in expression of pro-inflammatory cytokines) was evident from day 1 after TBI and ceased by day 14 , while in the contralateral hippocampus it was mainly limited to the dorsal part on day 1 . TBI induced an increase in hippocampal corticosterone level on day 3 bilaterally and an accumulation of II $1 \mathrm{~b}$ on day 1 in the ipsilateral hippocampus. Activation of microglia was observed from day 7 in different hippocampal areas of both hemispheres. Neuronal cell loss was detected in the ipsilateral dentate gyrus on day 3 and extended to the contralateral hippocampus by day 7 after TBI. The data suggest that TBI results in distant hippocampal damage (delayed neurodegeneration in the dentate gyrus and microglia proliferation in both the ipsilateral and contralateral hippocampus), the time course of this damage being different from that of the neuroinflammatory response.

\section{Introduction}

A leading cause of disability among young people is traumatic brain injury (TBI), a damage to the brain that occurs as a result of exposure to external mechanical force $[1,2,3]$. TBI can result in temporary or permanent impairments of cognitive and motor functions as well as the development of other neurological complications $[1,4]$. The prognosis of the disease depends on the nature, localization, and severity of the injury[4, 5].

Regardless of the injury severity, the delayed period after TBI in lots of patients is associated with the development of comorbid cognitive disorders, down to dementia, as well as emotional disturbances, commonly depression [6], which are believed to be associated with the dysfunction of the hippocampus. The casual involvement of the hippocampus in posttraumatic brain pathology is rather unexpected in mild TBI since the damage is focal, does not directly affect hippocampus and, as a rule, has an obvious effect only in the cerebral cortex $[4,5]$. Deeper structures, including the hippocampus, are not directly damaged at the moment of injury, and their delayed damage is distant, relative to the region of primary damage. The TBI-induced distant damage may involve not only the ipsilateral, but also the contralateral hippocampus (see $[6,7]$ for review).

Mechanical damage of cerebral tissue during TBI results in the cell death and the release of molecules that activate and maintain neuroinflammatory cascades $[8,9]$. These events are accompanied by a change in the number and shape of microglial cells; activation of the synthesis of proinflammatory cytokines (IL-1b, IL-6, TNFa) and other inflammation-related paracrine factors (chemokines, prostaglandins, reactive nitrogen and oxygen species); disruption of the blood-brain barrier integrity and the recruiting of immune cells from the peripheral circulation. It is suggested that these out-of-control neuroinflammatory processes may underlie post-traumatic cognitive and emotional disturbances. 
Previous studies with experimental models of traumatic brain injury reported changes in protein concentration of proinflammatory cytokines such as IL-1b, IL-6 and TNFa, as well as the levels of gene expression of these cytokines in the neocortex and hippocampus. These studies were focused on the changes occurring in the injured cortical area [10-18]. Using various models of TBI, different groups have shown that the protein levels of IL-1b, IL-6 and TNFa increase rapidly after injury and remain elevated in the ipsilateral part of the cortex up to 5-7 days after TBI. In contrast, changes in the hippocampal tissue are much less studied and, therefore, less understood.

Using a model of controlled cortical injury, it was shown that the local cortical trauma results in changes of cytokine expression in the ipsilateral hippocampus. An increase in the protein level of IL-1b on day 21 [19], IL-6 on days 3 and 21 [19, 20], as well as TNFa on days 2 and 21 after TBI [12, 19] were reported. Similarly, an increase in the concentrations of the IL-1b mRNA and protein in the entire hippocampus 3, 8, 12,48 hours after a diffuse brain injury was detected in the Marmarou's weight-drop model of TBI [21, 22]. Similar changes were observed in studies using the lateral fluid-percussion model of TBI [17, 18]. In these experiments, a rapid increase in Tnf and $\| 1 \mathrm{~b}$ mRNA levels and in respective proteins was observed in both the ipsilateral and contralateral regions of the cortex and hippocampus 1, 3, 24 and 72 hours after TBI. It was demonstrated that in the ipsilateral hemisphere a sharp increase in the level of both cytokine mRNA expression and the respective protein occurred during the very early period after the injury (1h and $3 \mathrm{~h})$, and in 24 hours the levels of these cytokines return to those of sham-operated animals. In the contralateral hemisphere, the changes were also present, though they were less pronounced: a slight reversible increase in the concentration of TNFa (both in the cortex and in the hippocampus) and IL-1b (in the hippocampus only). In different TBI models, these changes in the cytokine concentrations were accompanied by changes in the state of microglia at different time points, not only in the neocortex [14, $20,23,24]$, but also in the hippocampus [14, 20, 25].

It should be noted that the hippocampus is highly heterogeneous, both structurally and functionally. Regarding septo-temporal gradient of this structure, two main regions are usually studied, the dorsal hippocampus and the ventral hippocampus [7, 26]. Different involvement of the dorsal and ventral hippocampus in the realization of different brain functions may be potentially associated with different changes occurring in these hippocampal areas after TBI. However, a detailed analysis of the changes occurring in the dorsal and ventral hippocampus after TBI has not been performed. In addition, from the perspective of understanding, predicting and treating the neuroinflammation-related consequences of $\mathrm{TBI}$, one of important factors may be the proximity of the hippocampus to the damaged area of the neocortex. However, this issue was entirely neglected in previous studies.

In the present study, we investigated the time course of changes in the levels of gene expression associated with the neuroinflammation and stress reactivity in the dorsal and ventral hippocampus, corticosterone and proinflammatory cytokines concentrations in the dorsal and ventral hippocampus and blood serum, as well as the hippocampal microglia state during early period after TBI.

\section{Materials And Methods}


All procedures were performed in accordance with the ARRIVE guidelines and the U.K. Animals (Scientific Procedures) Act, 1986 and associated guidelines, EU Directive 2010/63/EU for animal experiments, or the National Institutes of Health guide for the care and use of Laboratory animals (NIH Publications No. 8023, revised 1978). The experimental protocol was approved by the Ethics Committee of Institute of Higher Nervous Activity and Neurophysiology, Russian Academy of Sciences (protocol number 10, December 10, 2012). All efforts were made to minimize animals' suffering.

\section{Animals}

The study was performed on 146 male 6 months old Wistar rats (BW327-455 g; median $380 \mathrm{~g}$ ). The animals were purchased in the "Stolbovaya" Breeding Center (Moscow Region) and were housed in acrylic cages with spruce shavings provided as bedding, in the institutional vivarium. The rats were kept at $12 \mathrm{~h}$ : $12 \mathrm{~h}$ day cycle, with free access to food and water.

The animals were randomly divided into 3 groups: 69 rats received lateral fluid-percussion brain injury; 60 rats were subjected to a scull trepanation without a brain injury (sham operated group); 18 rats formed the intact control group without surgical manipulations.

\section{Experimental Protocol and Surgery}

All surgical procedures were performed under inhalational anesthesia with 2-3\% isoflurane. Scull trepanation was made in the right parietal bone (3-mm aperture, $A P=3 \mathrm{~mm}, \mathrm{~L}=3 \mathrm{~mm}$ ). The head of a Luertype injection needle was fixed at the margin of the trepanned aperture with cyanoacrylate glue. Lateral fluid percussion brain injury $(3.03 \pm 0.03 \mathrm{~atm})$ was applied after recovery from anesthesia using a Fluid Percussion Device with the PC-Based Pressure Measurement Unit (Model FP302, USA). After TBI, the animals were returned to their home cages.

For biochemical analysis, the animals were sacrificed by decapitation using a guillotine, on days 1 (sham, $\mathrm{n}=9$; TBI group, $\mathrm{n}=8), 3$ (10 and 7 rats, respectively), 7 (11 and 7, respectively) and 14 (10 and 14, respectively). The brain was removed and briefly cooled in ice-cold saline. The hippocampus was isolated, and its ipsilateral and contralateral parts were divided into ventral and dorsal portions. Decapitation blood was sampled and centrifuged at $1500 \mathrm{~g}$ and $4^{\circ} \mathrm{C}$ for $15 \mathrm{~min}$ to obtain serum. For histological analysis, the rats were sacrificed under isoflurane anesthesia by the arterial perfusion with $4 \%$ formaldehyde solution in $0.1 \mathrm{M}$ phosphate buffer, $\mathrm{pH} 7.4$, on days 3 ( 11 shams and 8 rats from TBI group) and 7 ( 9 and 12 rats, respectively). The control groups for biochemical and histological analysis $(\mathrm{n}=9$ and 8 rats, respectively) were sacrificed similarly to respective experimental groups, and the biological material sampled accordingly.

\section{Histology and Morphometry}

Fifty- $\mu \mathrm{m}$ sections of rat brains were prepared using a vibratome. Sections located $600 \mu \mathrm{m}$ apart with coordinates between 2.1 and $5.8 \mathrm{~mm}$ from the bregma were selected for further analysis. The sections 
were stained using the Nissl method. Immunohistochemical staining for ionized calcium-binding adaptor molecule 1 (Iba1), a microglial marker, was performed using polyclonal rabbit-anti-lba1 (Dako, Denmark) diluted 1:500 with secondary antibodies (goat anti-rabbit IgG, Alexa Fluor, USA) diluted 1:1500. Microphotographs of the sections stained by the Nissl method were made using a Keyence BZ-X700 microscope (magnification x20). Microphotographs of immunohistochemically stained sections were made using a ZEISS Apotome microscopy (magnification $\times 20$ ). Further calculations of microglial cells were made using ImageJ program. Microglial cells in Iba-stained sections and neurons in Nissl-stained sections were counted in the field $150 \times 150 \mu \mathrm{m}$ in the polymorphic layer of the dentate gyrus (DG), CA1 and CA3 areas of the hippocampus. Representative microphotographs used for morphological descriptions and cell counts are presented in Fig. S1 (Nissl staining) and Fig. S2 (Iba1 immunostaining of microglial cells in the hippocampal dentate gyrus).

\section{Tissue Homogenization}

The ipsilateral and contralateral dorsal and ventral hippocampal tissue samples were homogenized using a Potter homogenizer in a 10 -fold excess of cold buffer for homogenization $(0,1 \%$ NP-40, the protease inhibitor (Roche), phosphate-buffered saline (PBS) with 10 impacts of the pestle at a rotation speed of $1000 \mathrm{rpm}$. The homogenate was additionally triturated with a $1 \mathrm{ml}$ pipette, then $50 \mu \mathrm{l}$ was taken into an Eppendorf test tube with $500 \mu \mathrm{l}$ of the reagent for RNA isolation Qiazol (Qiagen). This part of the homogenate was used for PCR analysis. The rest of the homogenate was centrifuged $(16900 \mathrm{~g}, 15 \mathrm{~min}$, $4^{\circ} \mathrm{C}$ ). The supernatants were used to determine the levels of proinflammatory cytokines and corticosterone by ELISA. The aliquots were stored at $-80^{\circ} \mathrm{C}$ until use.

\section{Enzyme-linked Immunosorbent Assay (ELISA)}

To determine serum and hippocampal corticosterone levels kits for enzyme-linked immunosorbent assay (Kit Corticosterone for 96 tests, cat. no. EIA4164; DRG) were used; the kits allow to detect both free and bound corticosterone by a competitive ELISA method.

The levels of proinflammatory cytokines IL-1 $\beta, I L-6$, TNFa in the serum and the hippocampus of rats were measured by R\&D Systems Quantikine ELISA Kits according to the manufacturer's instructions (cat.№ SRLB00; cat.no. SR6000B; cat.no. SRTA00).

\section{RNA Extraction and Reverse Transcription}

RNA was isolated using Qiazol reagent according to the manufacturer's recommendations. Before cDNA synthesis, $1 \mu \mathrm{g}$ of RNA was treated with DNase I (DNase I, Thermo Fisher) according to the manufacturer's recommendations. Then RNA was used to synthesize cDNA using a mix of random decaprimer and oligo(dT)-primer by means of the MMLV RT Kit (Evrogen) in accordance with the manufacturer's recommendations.

\section{Quantitative Real-Time Polymerase Chain Reaction (PCR)}


The gene expression was analyzed using qPCRmix-HS SYBR+LowROX (Evrogen) in accordance with the manufacturer's recommendations by means of a quantitative PCR system CFX384 (Bio-Rad). Nucleotide sequences of primers used are shown in Table S1.

The relative quantity (RQ) of transcripts was assessed using the $2^{-\Delta \Delta C t}$ method, taking into account the efficiency of the reaction with respect to the expression of the Hprt2 and Ywhaz genes; the data in the graphs are presented as RQ.

\section{Statistical Analysis}

The variable values were not normally distributed and groups had different variances (according to Shapiro-Wilk and Levene tests). The significance of differences between the experimental groups was determined by the Mann-Whitney test in the Python 3.7 SciPy software package. The data on graphs are presented as box plots: bars represent medians, boxplots represent quartiles, whiskers represent Q1$1.5 * \mid \mathrm{QR}$ and $\mathrm{Q} 3+1.5 * \mid \mathrm{QR}$. For all groups, $\mathrm{n} \geq 6$.

\section{Results}

\section{Analysis of hippocampus morphology after TBI}

We found that lateral fluid percussion-induced TBI altered the tissue morphology in the DG, CA1 and CA3 areas of the hippocampus. The analysis of neuronal cell density revealed that TBI induced cell loss occurring in both the ipsilateral and contralateral DG without any obvious effects in CA1 and CA3 areas. The time course of cell loss in the contralateral and ipsilateral DG was different: in the ipsilateral part it was observed on day 3 (Fig. S3), while in the contralateral DG, it appeared only on day 7 after TBI (Fig. 1).

Neuronal degeneration may be potentially associated with microglial activation; therefore, we analyzed the status of microglia using Iba1 staining. An increase in the number of microglial cells became evident only on day 7 after TBI in both hemispheres in ventral and dorsal parts of the hippocampus (Fig. 2). Unexpectedly, neuronal cell death on day 3 in the ipsilateral dorsal hippocampus was not associated with microgliosis (Fig. S4).

To expand the data on the status of microglia, we performed measurements of changes in mRNA expression of microglial markers $\mathrm{C} \times 3 \mathrm{cr} 1$ and $\mathrm{Ncf} 1$, as well as fractalkine (Cx3cl1), a major neuronal cytokine regulating the state of microglia. No changes were observed in gene expression levels of the chemokine fractalkine $\mathrm{Cx} 3 \mathrm{cl} 1$ (Fig. S5) when we compared the TBI and sham-operated groups; however, in contralateral parts of the hippocampus and the ipsilateral dorsal hippocampus we found a decrease in fractalkine expression in sham-operated animals as compared to respective intact groups during first days after he operation. Similarly, the expression of fractalkine receptor also decreased on the first day in all hippocampal parts, except of the ipsilateral dorsal hippocampus as compared to both sham-operated and intact animals. Analyzing the effect of TBI, we observed a significant increase in Cx3cr1 expression in all hippocampal parts (Fig. S6) on the first day. In the contralateral hippocampi, this increase ceased by 
day 3 (Fig. S6 a, c) whereas in the ipsilateral hippocampus, it remained elevated for at least 7 days and declined to baseline on day 14 (Fig. S6 b, d). Changes in mRNA of another microglial marker, Ncf1, were similar to those of $\mathrm{Cx} 3 \mathrm{cr} 1$; TBI resulted in an increase in its expression which ceased by day 14, however, this increase was observed only in the ipsilateral hippocampus (Fig. S7). Thus, these data support morphological data showing long-term activation of microglia after TBI.

\section{TBI-induced changes in the level of stress-related markers}

Corticosterone is a well-known marker of stress response and, therefore, we measured its level in different hippocampal regions and blood serum. Corticosterone concentration increased in all parts of the hippocampus of the ipsilateral and contralateral hemispheres on day 3 after $\mathrm{TBI}$, and by day 7 it dropped to the level observed in sham-operated animals (Fig. 3). The time course of corticosterone level in the blood serum was the similar to that in the hippocampus (Fig. S8a).

It is known that the corticosterone binds to glucocorticoid and mineralocorticoid receptors for realizing its physiological response [6]. These receptors are abundant in the cells of the hippocampus, and changes in their expression and related signaling may reflect changes in the response of hippocampal cells to changes in corticosterone concentration. Therefore we have analyzed hippocampal mRNA expression of glucocorticoid (Nr3c1) and mineralocorticoid (Nr3c2) receptors. In addition, the stress response in the hippocampus may involve corticotropin-releasing hormone (Crh) signaling since some hippocampal interneurons synthesize $\mathrm{CRH}$, a peptide hormone involved in stress response, and express two types of its receptors (Crhr1 and Crhr2) are present on hippocampal cells. However, did not TBI induce significant changes in the expression of Crh (Fig. S9), Crhr1 (Fig. S10), Crhr2 (Fig. S11), Nr3c1 (Fig. S12) and Nr3c2 (Fig. S13).

\section{Time course of cytokine response after TBI}

First, we evaluated changes in the concentrations of IL-1b, IL-6 and TNFa in the blood serum after TBI. No changes in serum level of three cytokines measured could be revealed after TBI as compared with shamoperated groups (Fig. S8 b-d).

In contrast to the blood cytokines, expression of these cytokines in the hippocampus changed significantly both after TBI as compared with sham-operated animals and in sham-operated animals as compared with controls. It should be noted that the mRNA of $\| 1 \mathrm{~b}$ increased in sham-operated animals in both the ipsi- and contralateral hippocamusas compared to intact animals (Fig. 4). A stronger effect was observed in the dorsal hippocampus (Fig. 4 a, b) where trepanation induced an increase in II1b mRNA occurring on day 3 and remaining at a high level until day 14. The ventral hippocampus appeared to be less sensitive to trepanation since an increase in the Il1b mRNA was observed only on day 14 after the surgery (Fig. 4 c, d). The mRNA level of $I 16$ and Tnf was virtually insensitive to trepanation: we observed a statistically significant increase in Tnf mRNA only in the ipsilateral ventral hippocampus on day 14 (Fig. $5 d)$. 
TBI resulted in a pronounced increase in the mRNA expression of the $I 11 \mathrm{~b}, \mathrm{Tnf}$, and $\| 16$ in the ipsilateral dorsal and ventral hippocampus as well as in the contralateral dorsal hippocampus as compared to sham-operated animals. This response reached a maximum on day 1 after TBI. Further, level of the II1b

(Fig. 4) and Tnf (Fig. 5) genes expression decreased by day 14 to the level observed in sham-operated animals. The time course of 116 expression differed substantially from that of the above cytokines. II6 level decreased already on day 3 in all parts of the hippocampus, though it increased again in the ipsilateral dorsal hippocampus on day 7after the injury (Fig. 6).

We also measured protein levels of IL-1b and IL-6 in the hippocampal parts and found that the concentration of IL-1b significantly increased on day 1 after injury in the regions of the hippocampus in the ipsilateral hemisphere only (Fig. 7). The level of the IL-6 protein in the hippocampus was not affected by either sham operation or TBI (Fig. S14).

\section{Discussion}

During the last decades it became evident that focal brain injuries (particularly, most prevalent of them, TBI and stroke) induce distant ("secondary") damage of remote brain structures. In this regard, hippocampus is one of primary candidates for such a distant damage. Selective vulnerability of the hippocampus to different harmful factors has been well documented (see [7] for review). One of the hypotheses explaining the causes and mechanisms of remote hippocampal damage after focal injuries of other brain regions suggests that pathological changes to the hippocampus are glucocorticoiddependent [6]. According to this hypothesis, the excess of glucocorticoids secreted after a focal brain injury which is stressogenic by nature, interacts with corticosteroid receptors abundant in the hippocampus and activates signal transduction pathways which stimulate neuroinflammation. Subsequent events may include hippocampal neurodegeneration and disturbances in neurogenesis in the subgranular niche [32]. According to this hypothesis, over-activation of hypothalamic-pituitary-adrenal axis may be the central triggering event of remote hippocampal damage and subsequent dysfunction. The hippocampus, a complex heterogeneous brain structure, is integrating cognitive and emotional response, the dorsal part being mostly involved in learning and memory, while the ventral part - in stressresponse and emotions [33]. This is a strong reason why hippocampal dysfunction may underlie delayed brain disturbances, both neurological and psychiatric ones, induced by focal brain damage of other brain regions.

The long-term period after TBI, regardless of the severity of the damage and the preservation of integrity of deep brain structures, is often associated with comorbid dementia and depression, which are attributable to hippocampal dysfunction. These effects of TBI were also described in experimental animal models and are believed to be associated with remote hippocampal damage [27; 28]. In this study, using a lateral fluid percussion model of TBI, we show that cortical damage results in a gradual escalation of structural changes in the hippocampus, both ipsilaterally and contralaterally to cortical injury site. Cortical damage also induces microglial proliferation, a cellular hallmark of neuroinflammation, in the hippocampus. On day 3 after TBI, a decrease in the density of neurons in the 
DG was found only in the ipsilateral hippocampus. By day 7, neuronal cell loss spread to the hippocampus of the contralateral hemisphere. At this time point, it was accompanied by an increase in the number of microglial cells not only in the DG, where neurons undergo degeneration, but also in the CA3 and CA1 subfields. It is generally believed that neuroinflammation associated with microglial activation is a pre-requisite of neurodegeneration, however, this suggestion is not supported by our data. Moreover, vice versa, proliferation of microglia follows the initial neuronal cell loss. The delay between neuronal cell death and microgliosis in the DG as well as proliferation of microglia in CA3 area suggest that there may be some independent factors triggering delayed proliferation of microglia and neuronal cell death in the hippocampus. The fact that neurodegeneration first appears in the contralateral DG may indicate the involvement of acute physical effects of cortical trauma impact on subcortical structures. However, further spreading of neuronal cell loss to the contralateral hemisphere may be a sign of subsequent switching mechanisms of distant hippocampal damage.

It is known that brain trauma leads to activation of neuroinflammatory cascades in the hippocampus and release of pro-inflammatory cytokines [12, 17-22]. Here we confirm previous reports from other groups that neuroinflammation occurs with different intensity in different parts of both contra- and ipsilateral hippocampus $[17,18]$. Moreover, we extend previous data by showing that the time course of cytokine response is different not only in the ipsilateral and contralateral hippocampus but also depends on the septo-temporal gradient, differing in the dorsal and ventral parts of the hippocampus. Indeed, our data suggest that a complex multidimensional pattern of pro-inflammatory cytokine expression induced by TBI is likely to exist in the hippocampus. This pattern may be determined by the nature of the cytokine, specific hippocampal area, physical proximity to the impact region (the ipsilateral vs. contralateral hemisphere), and the position in the septo-temporal gradient (the ventral vs. dorsal part).

Interestingly, our measurements of $I 11 \mathrm{~b}$ and $I 16$ in the hippocampus at level of protein and mRNA revealed different time course of changes after TBI. There may be several reasons for this inconsistency. An increase in II1 b mRNA reflects activation of microglia and, possibly, perivascular macrophages in response to changes in the state of hippocampal tissue. The strong increase in mRNA level during the first day after TBI inevitably results in the elevation of protein level. However, at later stages, a probable increase in the protein level of IL-1b may be masked by a number of processes: (i) intensification of IL-1b turnover, while an increase in IL-1b is compensated by an enhanced protein consumption and degradation; and (ii) neuroinflammation developing in the hippocampus after TBI may be accompanied by an increase in the permeability of blood-brain barrier and stronger leakage of cytokines to blood flow. The latter suggests that the changes in mRNA expression reflect response of cells to TBI whereas the protein level is a more complex parameter affected by different factors, and an accurate interpretation of cytokine protein levels requires knowledge on the rate of their degradation and washout from the tissue.

The use of sham operated animals is believed to be a panacea providing a good surgery control in models of diseases including surgery stages. It would be more honest recognizing that the use of shams suggests that effects of a surgery used to model a human disease which in fact does not include surgery can be just easily subtracted from the complex changes in the experimental groups. This assumption is 
most likely erratic since physiological responses to different damage (e.g. craniotomy and brain trauma per se in modeling TBI) imply myriads of mechanisms at different levels, both common and specific. Thus, the approach just to subtract changes of sham groups from those of experimental groups (what we are actually doing when using shams) is imperfect, but so far the only possible one. Previously, it has been shown that modeling TBI by lateral fluid percussion, though remains a golden standard in the field, does nevertheless have some drawbacks complicating the interpretation of the results obtained. For example, it was shown that craniotomy (sham operation) resulted in a drastic increase in the blood level of corticosterone, while brain trauma itself did not induce any additional effects on this parameter in the acute period after TBI [29]. We further extended these observations by finding that trepanation by itself can cause long-lasting changes in the expression of some cytokines (Cx3cl1, $\| 1 \mathrm{~b}, \mathrm{Tnf}$ ) in the hippocampus without changes at morphological level. The alterations demonstrated in the sham operated animals may affect the resulting outcome of neuroinflammation after TBI. This finding may be very important for pharmacological studies when some drugs are applied to modulate neuroinflammation after TBI.

Finally, brain trauma is accompanied by a generalized stress response of the body, which activates the hypothalamic-pituitary-adrenal axis. This universal response to an injury accompanied by release of corticosterone in rodents (cortisol in humans), may be closely related with pro-inflammatory events both in the brain, and in the whole organism [6]. In a model of diffusion TBI induced by midline fluid percussion, it has been found that the TBI-induced increase in blood corticosterone level lasts for at least one day [30]. However, in lateral percussion model, the increase in blood level of corticosterone is transient and comparable to the increase induced by trepanation in shams [29]. Here, we measured the concentration of corticosterone in the decapitation blood and in the hippocampal tissue. In agreement with the data reported in [29], we observed no robust increase in blood or hippocampal corticosterone 1 day after TBI. Instead, the concentrations of corticosterone increased only on day 3 after TBI in the hippocampus of both hemispheres and in the blood, then they returned to the level of sham-operated animals. The nature of corticosterone elevation on day 3 is not clear, however, this increase in corticosterone level may be important for the loss of neuroinflammation processes regulation occuring after TBI. It has been known for many years that glucocorticoids, and corticosterone in particular, are able to suppress of inflammatory response, this feature ensuring the clinical use of corticosteroids as antiinflammatory agents. However, many studies have suggested that a prolonged increase in corticosterone can lead to its conversion from anti-inflammatory to pro-inflammatory, specifically inducing neuroinflammation in the hippocampus (see [31] for review). Therefore, the increase in blood corticosterone and corticosterone accumulation in the hippocampus on day 3 after TBI may be one of factors enhancing neuroinflammation at later stages after TBI and inducing adelayed activation of microglia in hippocampal areas.

In conclusion, the early period after lateral fluid-percussion-induced traumatic injury of the neocortex in rats is associated with a neuroinflammatory cytokine response, neuronal death and microglia proliferation in the hippocampus. However, the time course of these processes is different, depending not 
only on the time period after TBI, but also on the proximity to the impact area (ipsilateral or contralateral) and position along the septo-temporal axis (the dorsal or ventral parts of the hippocampus).

\section{Declarations}

Funding This work was supported by RFBR grants 19-315-90081 (NG, LT) and 19-015-00258 (NG, IK, MN).

Conflicts of interest The authors have no conflicts of interest to declare that are relevant to the content of this article.

Availability of data and material All data generated or analyzed during this study are included in this published article and supplementary information files. Primary datasets generated and/or analyzed during the current study are available from the corresponding author on reasonable request.

Code availability Not applicable.

Authors' contributions Study concept and design were elaborated by NG and IK. TBI modeling, biomaterial preparation, data collection and analysis were performed by IK, LT, SF, NS, AV, AB, MN, AK, YM, MO. The first draft of the manuscript was written by LT, IK, and AK. NG and AB supervised the study, read and accomplished the final version of the manuscript.

Ethics approval All experiments were conducted in compliance with the ARRIVE guidelines and the U.K. Animals (Scientific Procedures) Act, 1986 and associated guidelines, EU Directive 2010/63/EU for animal experiments, or the National Institutes of Health guide for the care and use of Laboratory animals (NIH Publications No. 8023, revised 1978) and the Ethics Committee of Institute of Higher Nervous Activity and Neurophysiology, Russian Academy of Sciences (protocol number 10, December 10, 2012).

Consent to participate Not applicable.

Consent for publication The manuscript contains no individual person's data in any form.

Acknowledgements All authors thank their institutes for the support and encouragements.

\section{References}

1. Capizzi A, Woo J, Verduzco-Gutierrez M (2020) Traumatic brain injury: an overview of epidemiology, pathophysiology, and medical management. Med Clin N Am 104:213-238. https://doi.org/10.1016/j.mcna.2019.11.001

2. Coronado VG, Xu L, Basavaraju SV, McGuire LC, Wald MM, Faul MD, Guzman BR, Hemphill JD (2011) Surveillance for traumatic brain injury-related deaths - United States, 1997-2007. MMWR Surveill Summ 60:1-32.

3. Grafman J, Salazar AM (2015) Traumatic brain injury, part I, Handbook of Clinical Neurology.Elsevier B.V. 
4. Oberholzer M, Müri RM (2019) Neurorehabilitation of traumatic brain injury (TBI): a clinical review. Med Sci 7:47. https://doi.org/10.3390/medsci7030047

5. Eapen B, Cifu D (2018) Rehabilitation after traumatic brain injury. Elsevier,St. Louis (MO)

6. Gulyaeva NV (2019) Biochemical mechanisms and translational relevance of hippocampal vulnerability to distant focal brain injury: the price of stress response. Biochemistry (Moscow) 84:1306-1328. https://doi.org/10.1134/S0006297919110087

7. Gulyaeva NV (2019) Functional neurochemistry of the ventral and dorsal hippocampus: stress, depression, dementia and remote hippocampal damage. Neurochem Res 44:1306-1322. https://doi.org/10.1007/s11064-018-2662-0

8. Gaetz M (2004) The neurophysiology of brain injury. Clin Neurophysiol 115:4-18. https://doi.org/10.1016/S1388-2457(03)00258-X

9. Schmidt OI, Heyde CE, Ertel W, Stahel PF (2005) Closed head injury-an inflammatory disease? Brain Research Reviews 48:388-399. https://doi.org/10.1016/j.brainresrev.2004.12.028

10. Lagraoui M, Latoche JR, Cartwright NG, Sukumar G, Dalgard CL, Schaefer BC (2012) Controlled cortical impact and craniotomy induce strikingly similar profiles of inflammatory gene expression, but with distinct kinetics. Front Neur 3:155. https://doi.org/10.3389/fneur.2012.00155

11. Dalgard CL, Cole JT, Kean WS, Lucky JJ, Sukumar G, McCullen DC, Pollard HB, Watson WD (2012) The cytokine temporal profile in rat cortex after controlled cortical impact. Front Mol Neurosci 5:6. https://doi.org/10.3389/fnmol.2012.00006

12. Kuwar R, Rolfe A, Di L, Xu H, He L, Jiang Y, Zhang S, Sun D (2019) A novel small molecular NLRP3 inflammasome inhibitor alleviates neuroinflammatory response following traumatic brain injury. $\mathrm{J}$ Neuroinflammation 16:81. https://doi.org/10.1186/s12974-019-1471-y

13. Chen G, Shi J, Jin W, Wang L, Xie W, Sun J, Hang C (2008) Progesterone Administration Modulates TLRs/NF-KB Signaling Pathway in Rat Brain after Cortical Contusion. Ann ClinLab Sci 38:65-74.

14. Zhao G, Wang Y, Li Y, Jiang Z, Sun L, Xi X, He P, Wang G, Xu S, Ma D, Ke K (2014) The neuroprotective effect of modified "Shengyu" decoction is mediated through an anti-inflammatory mechanism in the rat after traumatic brain injury. J Ethnopharmacol 151:694-703. https://doi.org/10.1016/j.jep.2013.11.041

15. Mukherjee S, Katki K, Arisi GM, Foresti ML, Shapiro LA (2011) Early TBI-induced cytokine alterations are similarly detected by two distinct methods of multiplex assay. Front Mol Neurosci 4:21. https://doi.org/10.3389/fnmol.2011.00021

16. Hiskens M, Vella R, Schneiders A, Fenning A (2021) Celecoxib in a Preclinical Model of Repetitive Mild Traumatic Brain Injury: Hippocampal Learning Deficits Persist with Inflammatory and Excitotoxic Neuroprotection. Trauma Care 1:23-37. https://doi.org/10.3390/traumacare1010003

17. Vitarbo EA, Chatzipanteli K, Kinoshita K, Truettner JS, Alonso OF, Dietrich WD (2004) Tumor Necrosis Factor a Expression and Protein Levels after Fluid Percussion Injury in Rats: The Effect of Injury Severity and Brain Temperature. Neurosurgery 55:416-425. https://doi.org/10.1227/01.NEU.0000130036.52521.2C 
18. Kinoshita K, Chatzipanteli K, Vitarbo EA, Truettner JS, Alonso OF, Dietrich WD (2002) Interleukin-1 $\beta$ Messenger Ribonucleic Acid and Protein Levels after Fluid-Percussion Brain Injury in Rats: Importance of Injury Severity and Brain Temperature. Neurosurgery 51:195-203. https://doi.org/10.1097/00006123-200207000-00027

19. Zhang W, Li B, Guo Y, Bai Y, Wang T, Fu K, Sun G (2015) Rhamnetin attenuates cognitive deficit and inhibits hippocampal inflammatory response and oxidative stress in rats with traumatic brain injury. Centr Eur J Immunol 40:35-41. https://doi.org/10.5114/ceji.2015.50831

20. Gatson JW, Liu M, Abdelfattah K, Wigginton JG, Smith S, Wolf S, Minei J (2013) Resveratrol decreases inflammation in the brain of mice with mild traumatic brain injury. J. Trauma and Acute Care Surgery 74:470-475. https://doi.org/10.1097/TA.0b013e31827e1f51

21. Lu K, Wang Y, Yang J, Yang Y, Chen H (2005) Effect of Interleukin-1 on Traumatic Brain InjuryInduced Damage to Hippocampal Neurons. J Neurotrauma 22:885 - 895. https://doi.org/10.1089/neu.2005.22.885

22. Chao PK, Lu KT, Jhu JY, Wo YP, Huang TC, Ro LS, Yang YL (2012) Indomethacin protects rats from neuronal damage induced by traumatic brain injury and suppresses hippocampal IL-1 $\beta$ release through the inhibition of Nogo-A expression. J Neuroinflammation 9:121. https://doi.org/10.1186/1742-2094-9-121

23. Mei Z, Zheng P, Tan X, Wang Y, Situ B (2017) Huperzine A alleviates neuroinflammation, oxidative stress and improves cognitive function after repetitive traumatic brain injury. Metab Brain Dis 32:1861-1869. https://doi.org/10.1007/s11011-017-0075-4

24. Witcher KG, Dziabis JE, Bray CE, Gordillo AJ, Kumar JE, Eiferman DS, Godbout JP, Kokiko-Cochran ON (2020) Comparison between midline and lateral fluid percussion injury in mice reveals prolonged but divergent cortical neuroinflammation. Brain Res 1746:146987. https://doi.org/10.1016/j.brainres.2020.146987

25. Aungst SL, Kabadi SV, Thompson SM, Stoica BA, Faden Al (2014) Repeated mild traumatic brain injury causes chronic neuroinflammation, changes in hippocampal synaptic plasticity, and associated cognitive deficits. J Cer BI Flow \& Metab 34:1223-1232. https://doi.org/10.1038/jcbfm.2014.75

26. Fanselow MS, Dong HW (2010) Are the Dorsal and Ventral Hippocampus Functionally Distinct Structures? Neuron 65:7-19. https://doi.org/10.1016/j.neuron.2009.11.031

27. Hicks RR, Smith DH, Lowenstein DH, Saint Marie R, Mclntosh TK (1993) Mild experimental brain injury in the rat induces cognitive deficits associated with regional neuronal loss in the hippocampus. J Neurotrauma 10:405-414. https://doi.org/10.1089/neu.1993.10.405

28. Shultz SR, Bao F, Omana V, Chiu C, Brown A, Cain DP (2012) Repeated Mild Lateral Fluid Percussion Brain Injury in the Rat Causes Cumulative Long-Term Behavioral Impairments, Neuroinflammation, and Cortical Loss in an Animal Model of Repeated Concussion. J Neurotrauma 29:281-294. https://doi.org/10.1089/neu.2011.2123 
29. Grundy PL, Harbuz MS, Jessop DS, Lightman SL, Sharples PM (2001) The Hypothalamo-PituitaryAdrenal Axis Response to Experimental Traumatic Brain Injury. J Neurotrauma 18:1373-1381. https://doi.org/10.1089/08977150152725669

30. Rowe RK, Ortiz JB, Thomas TC (2020) Mild and Moderate Traumatic Brain Injury and Repeated Stress Affect Corticosterone in the Rat. Neurotrauma Reports 1:113-124. https://doi.org/10.1089/neur.2020.0019

31. Bolshakov AP, Tret'yakova LV, Kvichansky AA, Gulyaeva NV (2021) Glucocorticoids: Dr. Jekyll and Mr. Hyde of hippocampal neuroinflammation. Biochemistry (Moscow) 86:156-167. https://doi.org/10.1134/S0006297921020048

32. Podgorny OV, Gulyaeva NV (2021) Glucocorticoid-mediated mechanisms of hippocampal damage: Contribution of subgranular neurogenesis. J Neurochem157:370-392. https://doi.org/10.1111/jnc. 15265

33. Gulyaeva NV (2015) Ventral hippocampus, Stress and Psychopathology: Translational implications. Neurochem J 9:85-94. https://doi.org/10.1134/S1819712415020075

\section{Figures}



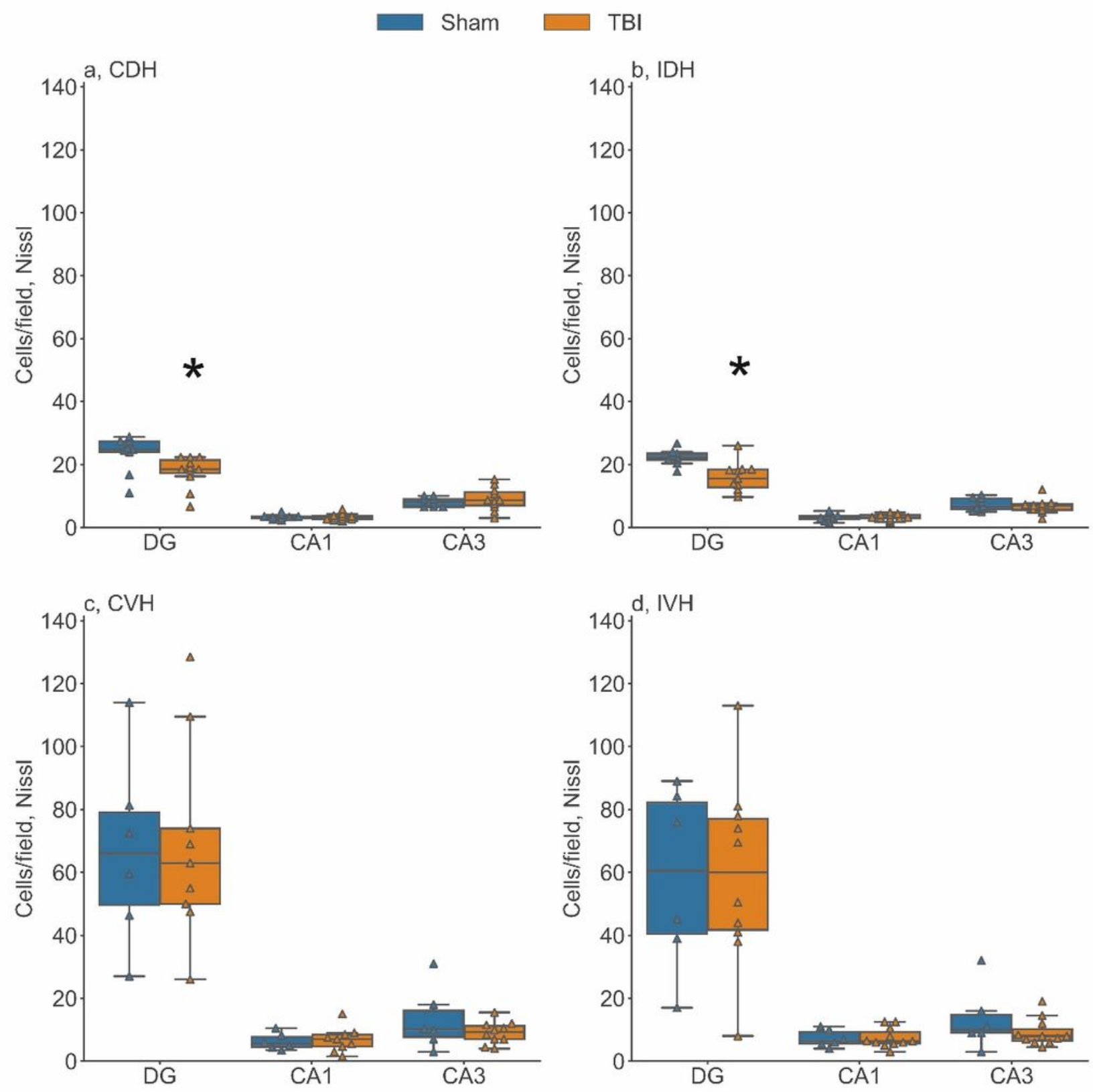

\section{Figure 1}

Nissl staining of neurons in hippocampal regions on day 7 after TBI. DG (dentate gyrus), CA1, CA3 hippocampal regions. The density of neurons in the DG of the dorsal hippocampus decreased bilaterally $(a, b)$ in TBI group as compared to shams. a Contralateral Dorsal Hippocampus (CDH); b Ipsilateral Dorsal Hippocampus (IDH); c Contralateral Ventral Hippocampus (CVH); d Ipsilateral Ventral Hippocampus (IVH) . * $\mathrm{p}<0.05$, Mann-Whitney U-test, TBI rats compared to Sham-operated rats 

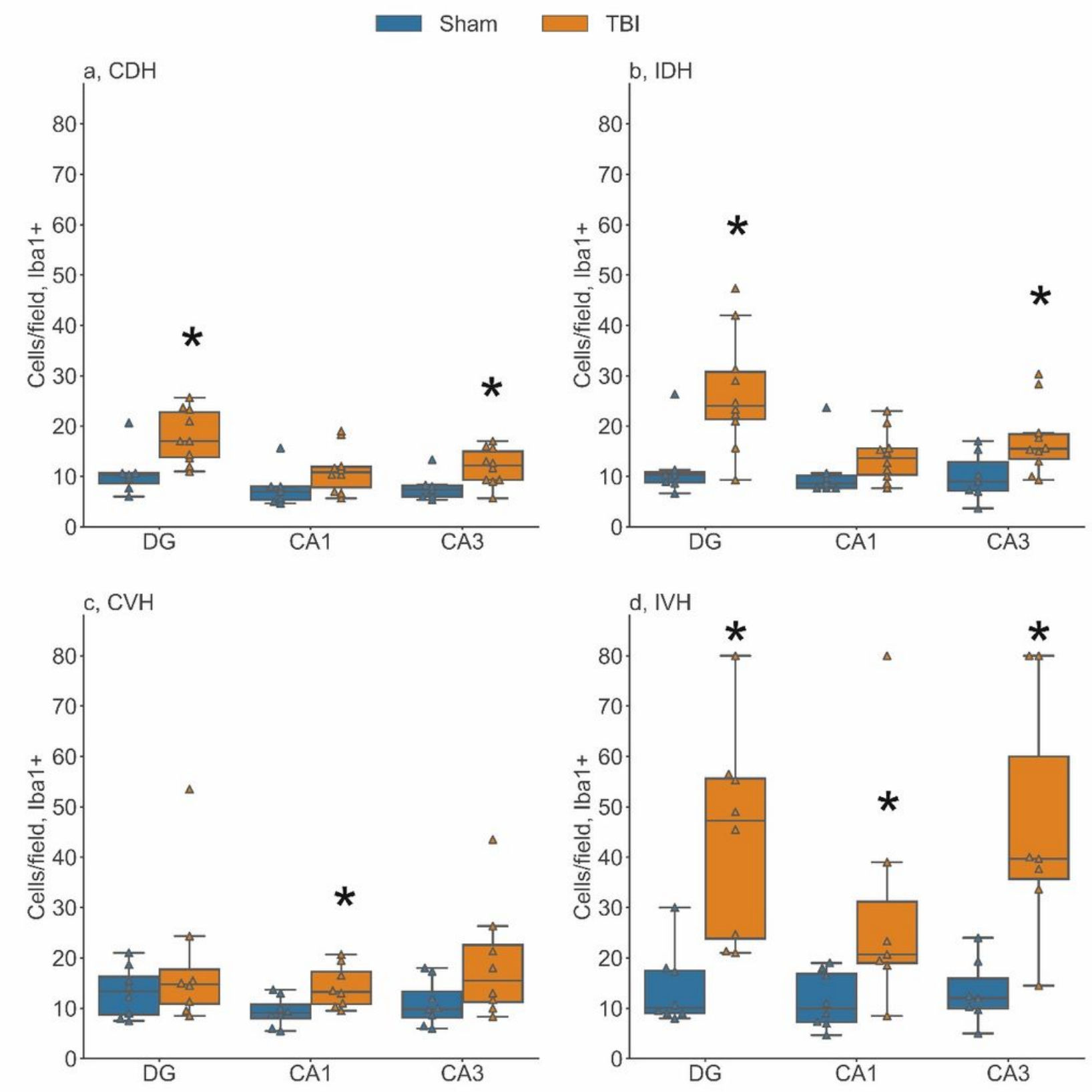

\section{Figure 2}

Iba1 immunostaining of microglial cells in hippocampal regions on day 7 after TBI. DG (dentate gyrus), CA1, CA3 - hippocampal regions. The number of microglial cells increased bilaterally in the dorsal and ventral hippocampus of rats with TBI. The number of microglial cells increased in CA3 areas of the DH (a, b) and VH (c, d), DG of IDH (b) and CDH (a) and CA1 areas of IVH (d) and CVH (c). a Contralateral Dorsal Hippocampus (CDH); b Ipsilateral Dorsal Hippocampus (IDH); c Contralateral Ventral Hippocampus 
(CVH); d Ipsilateral Ventral Hippocampus (IVH). * - $p<0.05$, Mann-Whitney U-test, TBI rats compared to Sham-operated rats. \# - $p<0.05$, Mann-Whitney U-test, Sham-operated rats compared to Intact rats

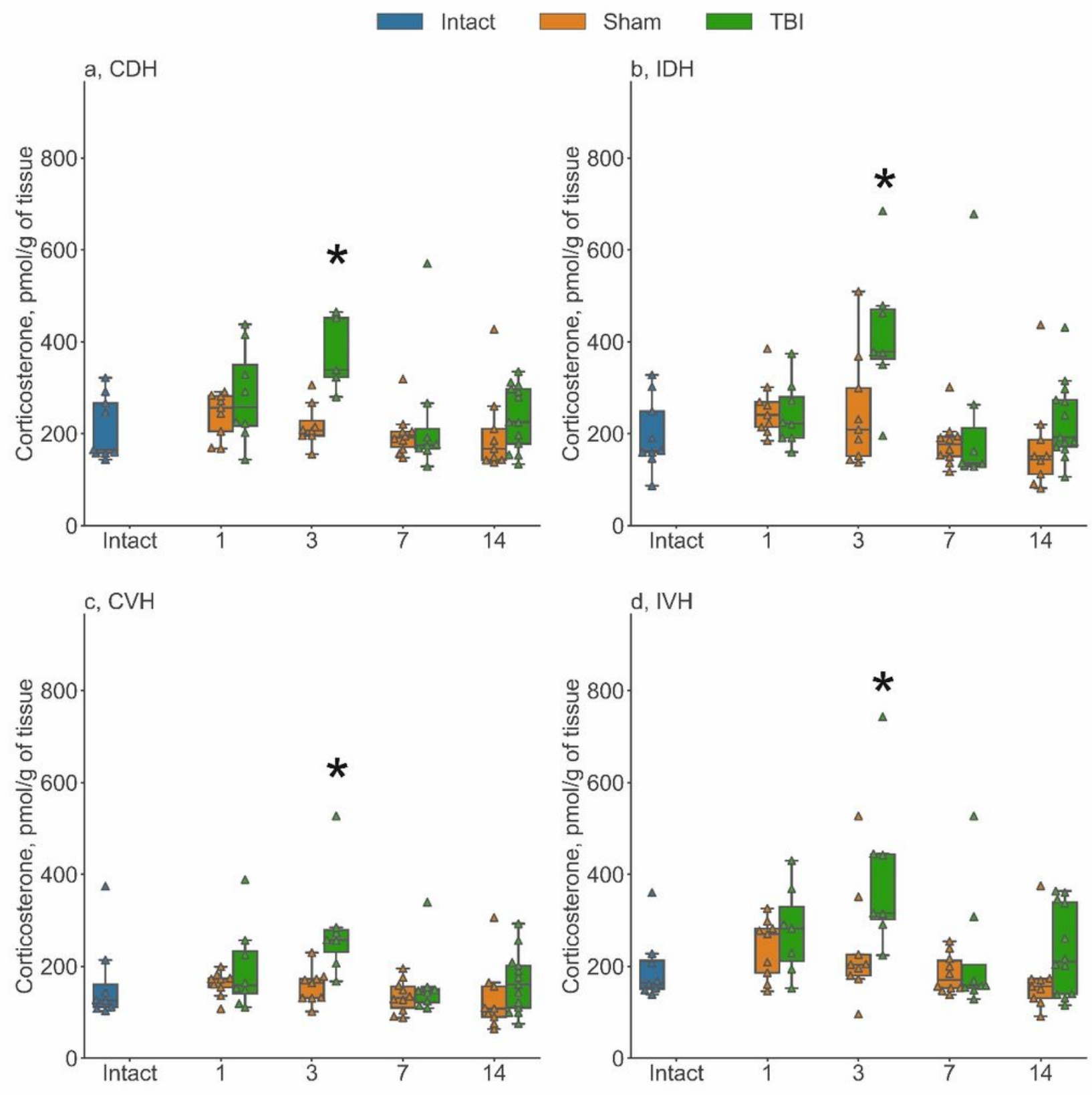

\section{Figure 3}

Changes in corticosterone concentration in different regions of the hippocampus in early period after lateral fluid percussion TBI (1-14 days). TBI induced an increase in corticosterone concentration only on day 3 in all hippocampal regions studied. a Contralateral Dorsal Hippocampus (CDH); b Ipsilateral Dorsal 
Hippocampus (IDH); c Contralateral Ventral Hippocampus (CVH); d Ipsilateral Ventral Hippocampus (IVH). * - $p<0.05$, Mann-Whitney U test

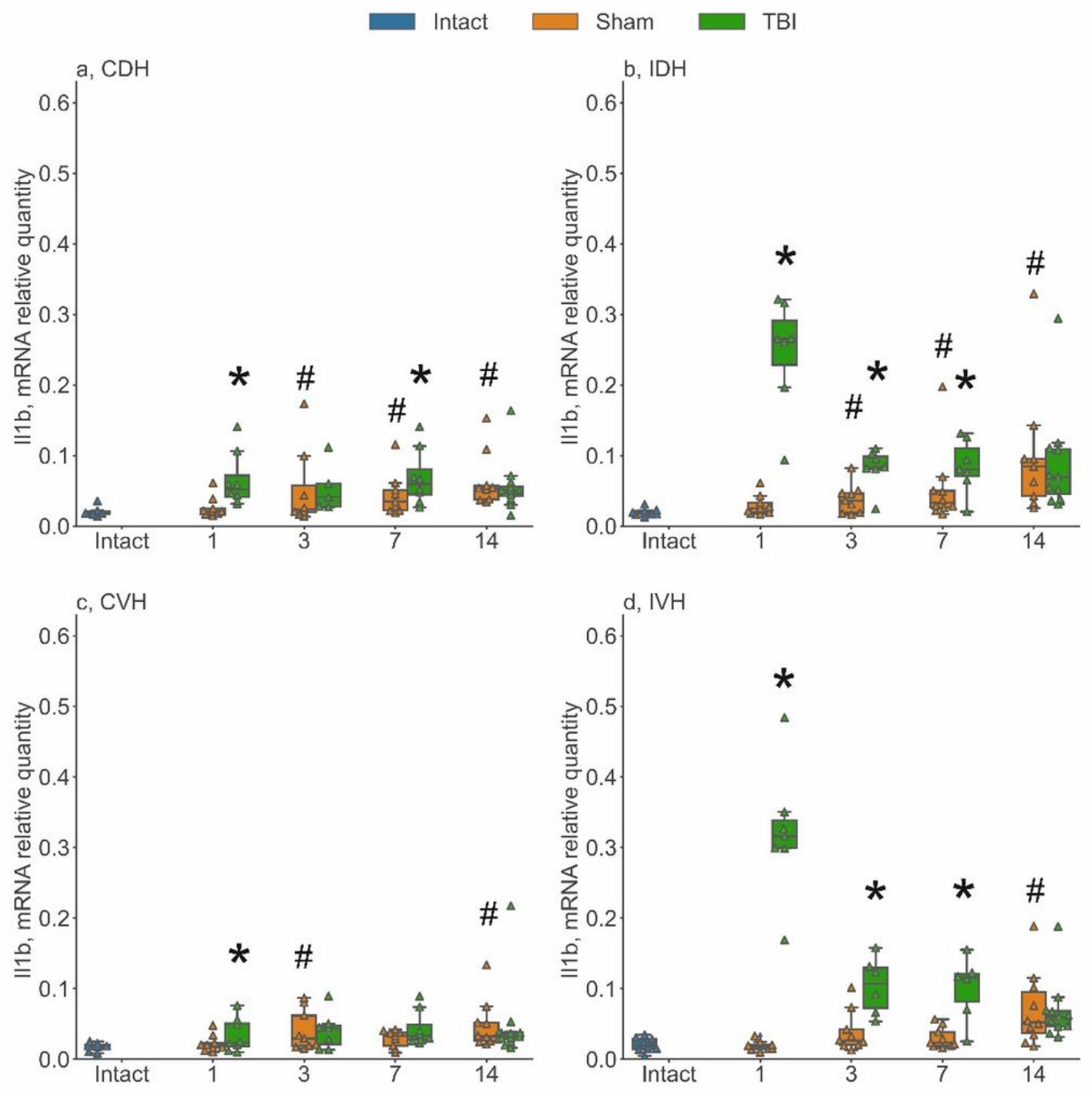

Figure 4

The time course of $I 11 \mathrm{~b}$ mRNA expression in different regions of the hippocampus in early period after lateral fluid percussion TBI (1-14 days). TBI induced an increase in II1b expression in the ipsilateral dorsal and ventral hippocampus on day 1 , then it gradually declined to the level of sham-operated animals on day $14(b, d)$. The hippocampus of the contralateral hemisphere responded by a small increase in II $1 \mathrm{~b}$ 
expression on day $1(\mathrm{a}, \mathrm{c})$ followed by the second wave of increase in the dorsal hippocampus on day 7 (a). Note that in sham-operated animals, an increase in the II1b expression in the ipsilateral dorsal hippocampus (b) and contralateral dorsal hippocampus (a) was observed on days 3, 7 and 14, in the contralateral ventral hippocampus on the days 3 and 14, and in the ipsilateral ventral hippocampus only on day 14 , as compared to intact animals. a Contralateral Dorsal Hippocampus (CDH); b Ipsilateral Dorsal Hippocampus (IDH); c Contralateral Ventral Hippocampus (CVH); d Ipsilateral Ventral Hippocampus (IVH). * - p<0.05, Mann-Whitney U-test, TBI rats compared to Sham-operated rats. \# $p<0.05$, Mann-Whitney U-test, Sham-operated rats compared to Intact rats

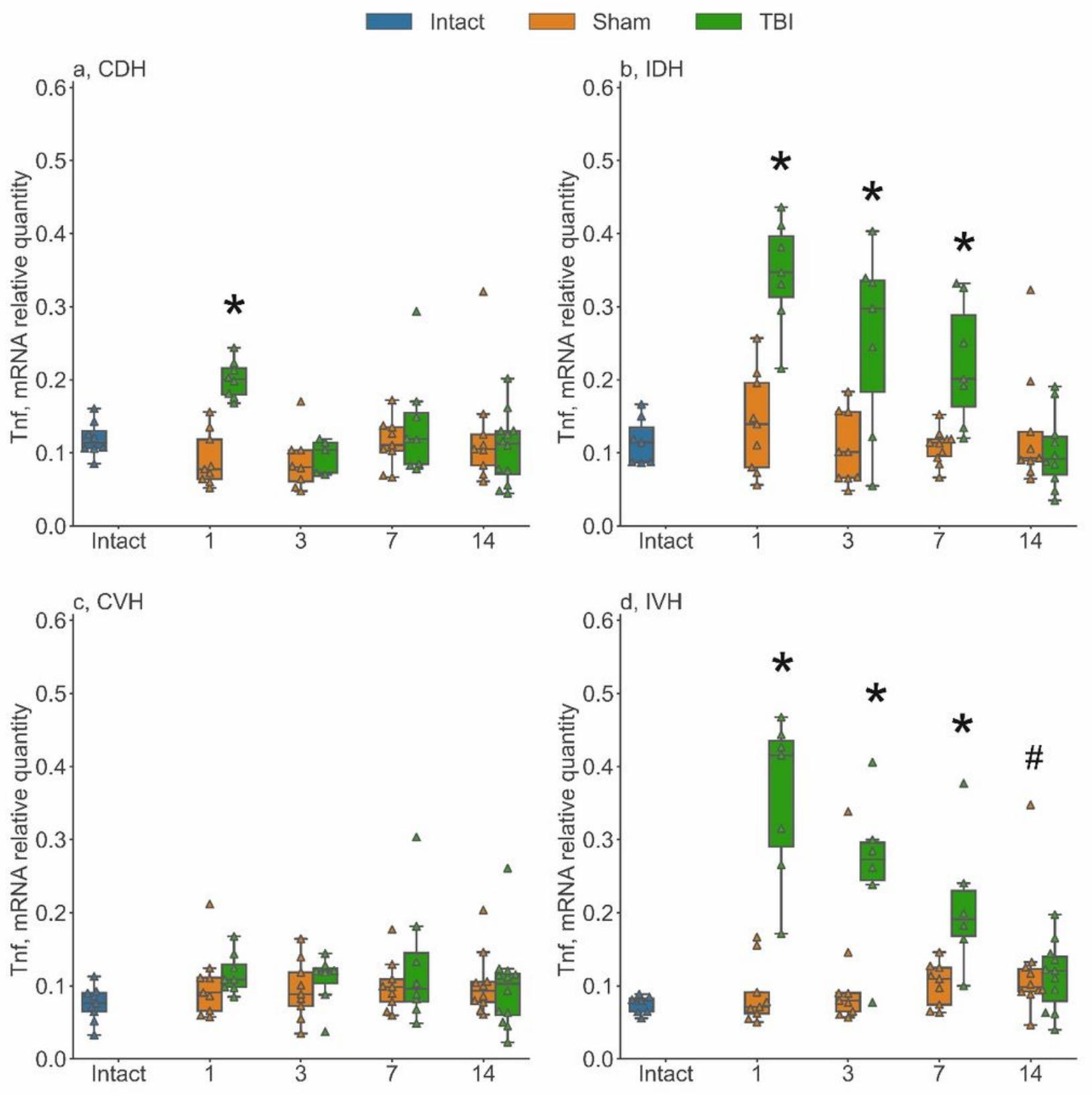




\section{Figure 5}

The time course of Tnf mRNA expression in different regions of the hippocampus in early period after lateral fluid percussion TBI (1-14 days). TBI induced an increase in Tnf expression in the ipsilateral dorsal and ventral hippocampus on day 1 , which gradually declined to the level of sham-operated animals by day 14 ( $b$ and $d)$. In the contralateral hemisphere, only the dorsal hippocampus responded by a small increase in Tnf expression on day 1 (a) whereas, in the ventral hippocampus, no changes were observed (c). Note that in sham-operated animals, we also observed an increase in the expression in the ipsilateral ventral hippocampus on day 14 as compared to intact animals. a Contralateral Dorsal Hippocampus (CDH); b Ipsilateral Dorsal Hippocampus (IDH); c Contralateral Ventral Hippocampus (CVH); d Ipsilateral Ventral Hippocampus (IVH). * $\mathrm{p}<0.05$, Mann-Whitney U-test, TBI rats compared to Sham-operated rats at respective day. \# - $p<0.05$, Mann-Whitney U-test, Sham-operated rats compared to Intact rats 

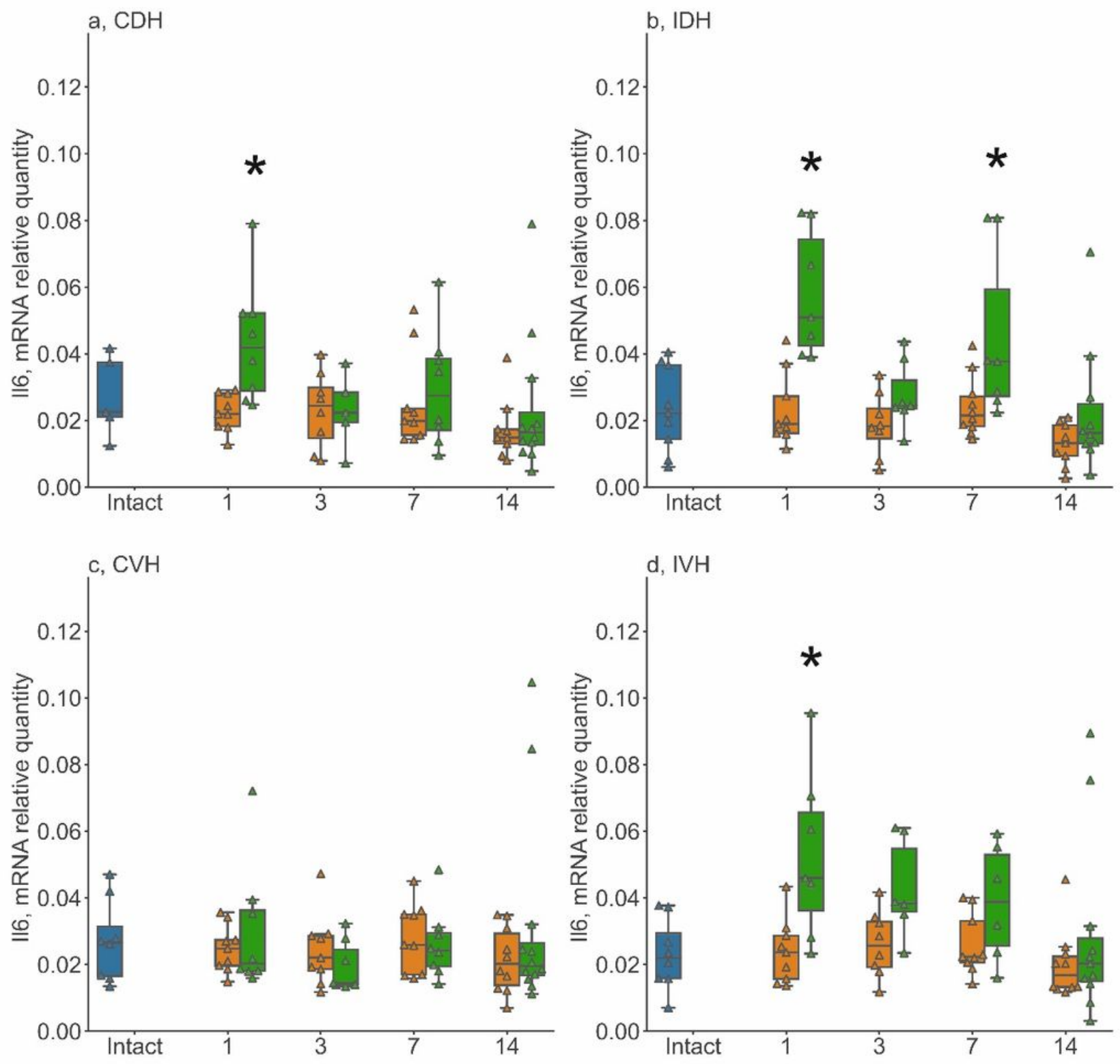

\section{Figure 6}

The time course of 116 mRNA expression in different regions of the hippocampus in early periodafter lateral fluid percussion TBI (1-14 days). TBI induced an increase in II6 expression on day 1 in the ipsilateral dorsal and ventral (b, d) and contralateral dorsal hippocampus (a); later it returned to the expression level similar to that of the respective sham-operated group. The II6 expression in the ipsilateral dorsal hippocampus (b) demonstrated the second increase on day 7 after TBI. In the contralateral ventral hippocampus, no changes were observed (c). a Contralateral Dorsal Hippocampus (CDH); b Ipsilateral 
Dorsal Hippocampus (IDH); c Contralateral Ventral Hippocampus (CVH); d Ipsilateral Ventral Hippocampus (IVH). * - p<0.05, Mann-Whitney U-test, TBI rats compared to Sham-operated rats. \# $\mathrm{p}<0.05$, Mann-Whitney U-test, Sham-operated rats compared to Intact rats

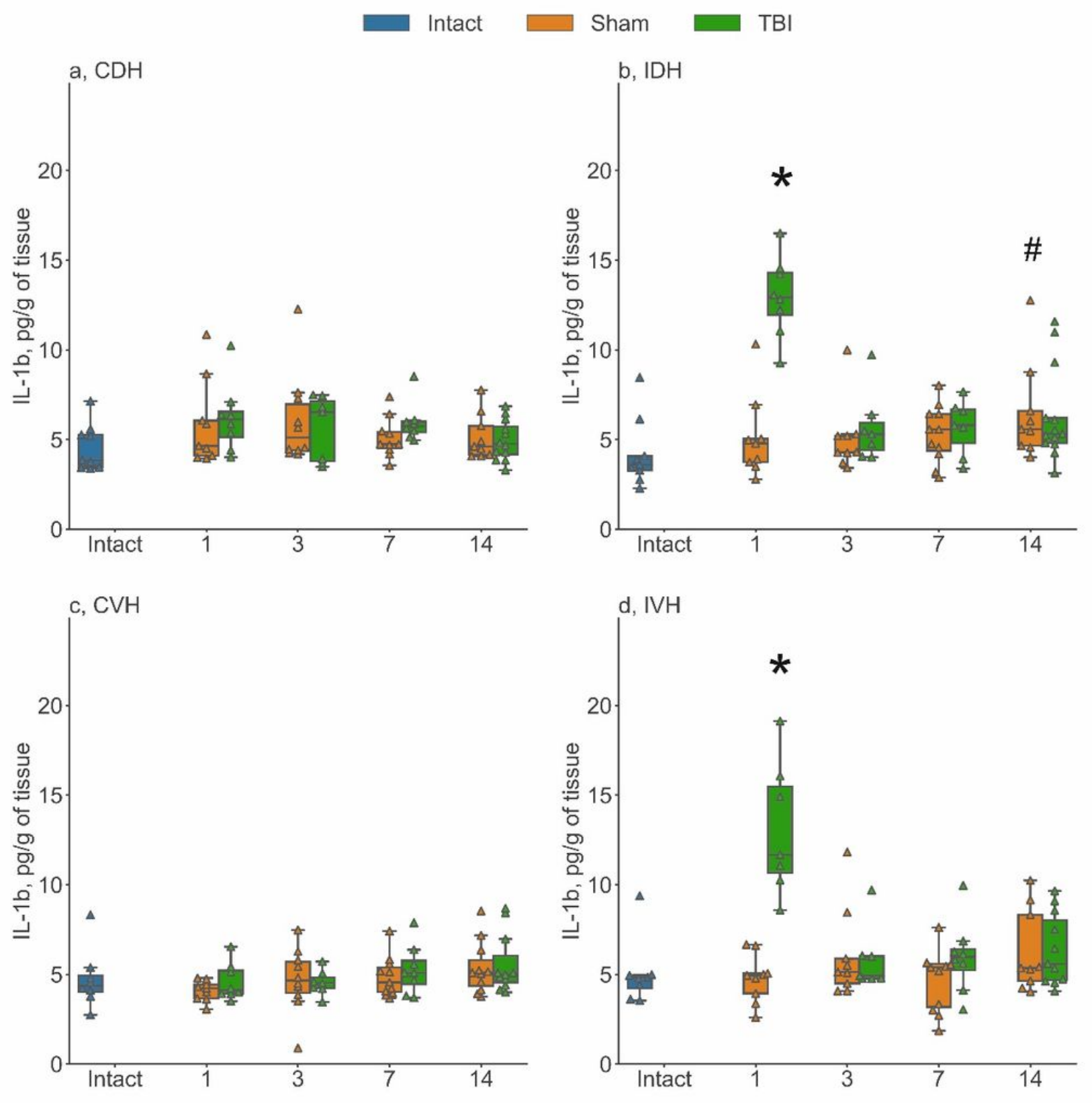

\section{Figure 7}

Changes in IL-1b concentration in different regions of the hippocampus in early period after lateral fluid percussion TBI (1-14 days). TBI induced an accumulation of IL1b on day 1 in the ipsilateral dorsal and ventral hippocampus $(b, d)$. In the contralateral hippocampus no changes could be revealed $(a, c)$. The 
accumulation of IL $1 \mathrm{~b}$ was also evident on day 14 in the ipsilateral dorsal hippocampus of sham-operated rats. a Contralateral Dorsal Hippocampus (CDH); b Ipsilateral Dorsal Hippocampus (IDH); c Contralateral Ventral Hippocampus (CVH); d Ipsilateral Ventral Hippocampus (IVH). * - p<0.05, Mann-Whitney U-test, TBI rats compared to Sham-operated rats. \# - $p<0.05$, Mann-Whitney U-test, Sham-operated rats compared to Intact rats

\section{Supplementary Files}

This is a list of supplementary files associated with this preprint. Click to download.

- SupplementaryInformationMolNeurobiol2021 revised.docx 